\title{
La calidad de la educación como parte del problema \\ Educación escolar y desigualdad en Chile
}

\author{
EDUARDO CAVIERES FERNÁNDEZ
}

Universidad de Playa Ancha, Valparaíso, Chile

\section{RESUMEN}

E1 sistema educativo chileno se encuentra en crisis debido a la desigualdad originada por una privatización que ha conducido a la segregación, con la consiguiente exclusión de los estudiantes de escasos recursos económicos. No obstante, existe cierto grado de consenso social en lo referente a mejorar la calidad educativa en el país. En el presente artículo se discute críticamente esta idea de que la mejora de la calidad educativa en los establecimientos escolares es parte de la solución a la crisis del sistema chileno. Contrariamente a esa idea, se plantea que, en realidad, esta calidad es parte del problema. Para desarrollar este argumento, el artículo se estructura en torno a tres planteamientos fundamentales: que la calidad educativa es parte de la privatización de los centros escolares; es parte de la segregación que se produce entre ellos, y es parte de la exclusión que perjudica a los alumnos de los sectores más empobrecidos económicamente del país.

PALABRAS CLAVE

desigualdad; privatización; calidad de la educación. 


\title{
QUALITY EDUCATION AS PART OF THE PROBLEM. SCHOOL EDUCATION AND INEQUALITY IN CHILE
}

\begin{abstract}
The chilean educational system is in crisis due to the inequality caused by privatization that led to segregation, with the exclusion of low-income students as a consequence. Nevertheless, there is a certain level of social consensus around the need to improve the quality of education in the country. This paper discusses critically the idea that the improvement of the education quality in schools is part of the solution to the crisis in the educational system. On the contrary, this quality is part of the problem. To develop this argument, the paper is structured around three main statements: that the quality of education is part of the privatization of schools, is part of the resulting segregation, and is part of the exclusion that harms the low-income students of the country.
\end{abstract}

KEYWORDS

inequality; privatization; quality of education.

\section{A QUALIDADE DA EDUCAÇÃO COMO PARTE DO PROBLEMA. EDUCAÇÃO ESCOLAR E DESIGUALDADE NO CHILE}

\section{RESUMO}

O sistema educativo chileno encontra-se em crise em razão da desigualdade originada por uma privatização que levou à segregação, com a consequente exclusão dos estudantes de escassos recursos econômicos. No entanto, existe certo grau de consenso social relativo a melhorar a qualidade educativa no país. No presente artigo, discute-se criticamente a ideia de que a melhora da qualidade educativa nos estabelecimentos escolares é parte da solução da crise do sistema chileno e propõe-se, em sentido contrário, que a qualidade é parte do problema. Para desenvolver esse argumento, o artigo se estrutura ao redor de três proposições fundamentais: que a qualidade educativa é parte da privatização dos centros escolares; é parte da segregação que se produz entre eles; e é parte da exclusão que prejudica os alunos dos setores do país mais empobrecidos economicamente.

PALAVRAS-CHAVE

desigualdade; privatização; qualidade da educação. 


\section{INTRODUCCIÓN}

El sistema educativo escolar chileno se encuentra bajo una profunda crisis debido a los niveles de desigualdad que se han producido en su interior. La privatización del sistema escolar ha generado el surgimiento de distintos tipos de establecimientos privados, pero sin una adecuada implementación de mecanismos para garantizar a los establecimientos públicos un sistema de financiamiento igualitario (Cornejo, 2006). Como consecuencia, los alumnos de escasos recursos económicos, concentrados en establecimientos públicos empobrecidos, han quedado desprovistos de las mismas oportunidades educativas que alumnos en establecimientos financiados privadamente. Por tanto, los resultados educativos presentan brechas significativas de desigualdad, concentrándose los malos resultados en los establecimientos públicos. En definitiva, el sistema educativo escolar chileno presenta severas fallas referidas a la privatización, lo que lleva a la segregación entre estudiantes de acuerdo a su estatus social, con la consiguiente exclusión de los estudiantes de escasos recursos económicos.

Diversos grupos sociales liderados por esos estudiantes desfavorecidos han protestado contra las desigualdades, demandando cambios sustantivos en el diseño del sistema educativo (Rojas, 2012). No obstante, mientras estas movilizaciones se oponen a las políticas educativas en lo referente a la privatización, en la medida en que la mejora de los aprendizajes de los alumnos es un derecho para todo ciudadano, las mismas tienden a mostrar un grado de consenso con las autoridades educativas en lo referente a mejorar la calidad de la educación en el país (Centro de Investigación y Desarrollo de la Educación, 2008; Gutierrez; Caviedes, 2006). La expresión "calidad de la educación" ha venido a constituir un sinónimo de buena educación que puede contener múltiples dimensiones, suscitando, así, su aprobación social. Sin embargo, en el contexto de reformas educativas internacionales, la calidad de la educación tiene una connotación muy precisa. El Banco Mundial, que ha sido la agencia con mayor impacto técnico y económico en estas reformas (Samoff, 1996; Torres, 2002), afirma que una definición de calidad educativa debe incluir los resultados de los alumnos; y esta "orientación hacia los resultados significa que las prioridades en la educación son determinadas a través de un análisis económico, de la implementación de estándares y la medición de esos estándares" (The World Bank, 1995a, p. 94).

En este artículo se discute críticamente la afirmación de que la falta de calidad en la educación escolar es una de las causas de la crisis del sistema educativo chileno y que, por tanto, el aumento de esa calidad representaría la solución al problema educacional en el país. Más bien, en este trabajo se argumenta que la calidad educativa $-y$ la racionalidad que representa- constituye un componente esencial del modelo educacional aplicado en Chile y, que por tanto, debe considerarse como parte del problema de desigualdad y exclusión que afecta a la educación chilena.Para desarrollar ese argumento, el artículo se estructura en torno a tres planteamientos fundamentales: que la expresión calidad educativa sirve para justificar la excesiva privatización de los centros escolares; para aumentar la segregación que se produce entre centros escolares privados y públicos; y para ahondar la exclusión educativa de los alumnos de los sectores económicamente más empobrecidos del país. 


\section{LA CALIDAD DE LA EDUCACIÓN COMO PARTE DE LA PRIVATIZACIÓN}

La noción de calidad de la educación se anida en una concepción más amplia sobre el capital humano. De acuerdo a los teóricos fundacionales de esta teoría, existen actividades humanas que afectan el consumo y el ingreso monetario $\mathrm{y}$, por tanto, se requiere invertir en las mismas para integrar el factor humano como variable de crecimiento económico (Becker, 1964; Schultz, 1971). En términos generales, esta inversión consiste en capacitar técnicamente a los trabajadores por medio de diferentes formas de educación que produzcan una forma de capital más eficiente. En décadas recientes, organismos como la Organization for the Economic Cooperation and Development (OECD) han integrado esta teoría en su conceptualización de la economía, otorgándole al conocimiento un rol fundamental y estableciendo una relación mucho más estrecha entre la educación y el mercado (Olssen; Peters, 2005). En Chile, la teoría del capital humano ha sido avanzada por los investigadores que más han influído sobre las reformas educativas en curso. En un texto frecuentemente citado, Brunner y Elacqua (2003) afirman que las instituciones educativas deberían contribuir a aumentar el capital humano en el país, dado que los avances en Chile se han producido precisamente por la inclusión de individuos con este capital dentro del círculo virtuoso formado por mercados, tecnología y un conjunto de políticas públicas apropiadas.

En las últimas décadas, estas perspectivas sobre la educación como capital han sido integradas en las múltiples reformas educativas que se han implementado mundialmente. Aunque con matices diversos, lo central de estas propuestas es transformar los sistemas educativos en cuasi mercados que mejoren su eficiencia para, a su vez, aumentar el crecimiento económico de los países (Wolff; Moura Castro, 2005). Esta mejora global de los sistemas educacionales pasa por ofrecer una variedad de proyectos educativos que permitan a los padres ejercer el derecho de educar a sus hijos, lo que generaría presión sobre los establecimientos escolares para aumentar sus rendimientos académicos y poder competir para atraer estudiantes y mejorar sus matrículas (Hill, 1996; Hoxby, 1994). Un segundo aspecto del modeloaunque distinguible-es que la calidad educativa que entreguen esos establecimientos debería garantizarse por medio de agencias centralizadas que prescriban el currículo y midan los aprendizajes de los alumnos (González, 2005).

En Chile, una primera ola de cambios educativos se llevó a cabo por la dictadura militar que gobernó entre los años 1973 y 1990. De acuerdo a Beyer, Eyzaguirre e Fontaine (2000), el objetivo fue proveer de libertad de elección a los padres y promover la competencia al interior del sistema. Para ello, se creó una nueva política de financiamiento para los establecimientos educativos (Torche, 2005). Esta política contempló que el Estado subsidiara a estos establecimientos conforme el nivel de asistencia de los alumnos a las clases. Además, se permitió a los colegios privados solicitar este subsidio a cambio de no cobrar tarifas altas a sus alumnos, aunque pudiendo seleccionar a sus estudiantes. Como resultado, el sistema escolar quedó conformado fundamentalmente por establecimientos públicos, subvencionados privados y privados de elite, los que en vez de recibir el subsidio estatal continuaron cobrando tarifas mayores. Los gobiernos democráticos 
que sucedieron a la dictadura no revirtieron ese proceso de privatización del sistema escolar, puesto que se consideró parte del proyecto de modernización de la educación necesario para el crecimiento económico del país (Arellano, 2005). En vez de ello, los esfuerzos se concentraron en mejorar la equidad, la calidad y la eficiencia del sistema, para lo cual se firmaron dos proyectos con el Banco Mundial (The World Bank, 1995b).

En buena medida, el Sistema de Medición de Calidad de la Educación implementado en Chile (SIMCE), y que tiene expresión en sus pruebas estándares (Ministerio de Educación, 2003), ha sido consistente con la privatización en curso, en la medida en que los mejores resultados se producen en los establecimientos educacionales bajo administración privada, especialmente en aquellos que atienden a los sectores con mayores ingresos económicos o que concentran las mayores ventajas sociales. Así, en términos de "producto" educativo medido en pruebas estándares -y no de "procesos" educativos-, la brecha en puntajes entre colegios privados, de provisión mixta y municipal ha sido enorme y consistentemente desigual (Gobierno de Chile, 2012; Universidad de Chile, 2011). En ese contexto, esta desigualdad ha impactado el sentido común de la población, que ve la calidad educativa como parte de la oferta contenida en la educación privada y que no está presente en la educación pública (Arancibia, 1994). Como resultado, en el año 2009, los establecimientos públicos dejaron de constituir el 64\% del total de establecimientos educativos en el país, como ocurría veinte años antes, y pasaron a constituir solo un 48\%; mientras que los establecimientos privados (establecimientos particulares subvencionados y privados de élite) pasaron de un $34 \%$ a un $52 \%$ del total de las unidades educativas (Departamento de Estudios y Desarrollo, 2010).

La calidad de la educación como racionalidad también es funcional a la privatización, ya que no exige que la misma deba ser detenida o regulada. Por lo contrario, la calidad de la educación y la privatización que conlleva se presenta como compatible con cualquier principio de igualdad social, ya que lleva integrado un componente inclusivo que también pone acento en la educación de los más desaventajados económicamente. Ello justificaría la afirmación que aumentar la calidad de la educación termina fortaleciendo tanto al sector público como al privado. Esta perspectiva ha sido sintetizada por Beyer (2009, p. 121), que en el contexto de la discusión sobre cómo mejorar el financiamiento de la educación pública afirma que "la discusión sobre la institucionalidad en la que debe apoyarse la educación pública es irrelevante al lado de los verdaderos desafíos que ésta tiene para avanzar en términos de calidad". La posición del autor es significativa por su prestigio como investigador y por haber sido ministro de educación en Chile. Esta posición es, además, consistente con la postura manifestada por los principales investigadores de educación en el país, en términos de que cualquier mejora que se introduzca al sistema debe contextualizarse dentro de las metas de una educación de calidad, lo que, a su vez, estaría en consonancia con la evidencia internacional (Belleï 2007; Brunner; Peña, 2007; Mizala, 2008). 
Actualmente, la OECD constituye probablemente la mayor referencia internacional en el país debido a que Chile fue integrado recientemente como miembro. De acuerdo a la OECD $(2004,2011)$, los déficits del sistema educativo chileno se deben a que se acentuaron excesivamente ciertos mecanismos privatizadores, tal como la competencia entre establecimientos educativos, sin que mejorara la eficacia y eficiencia en el sistema. No obstante, esto no significa que la OECD no reconozca méritos a la provisión mixta implementada, ya que ha permitido que los resultados de aprendizaje no decaigan a lo largo del tiempo y ha disminuido costos económicos para el Estado. También la OECD se inclina por reconocer que si el sistema chileno hubiese continuado formado mayoritariamente por colegios públicos tampoco hubiera ocurrido una mejora significativa en el desempeño de los alumnos. En este sentido, la OECD coincide con la postura de que la solución del sistema educativo chileno pasa fundamentalmente por acentuar la calidad de la educación. Por tanto, su principal crítica al sistema chileno es que ha habido insuficiente intervención estatal para garantizar estándares de aprendizaje y que, por tanto, su mejora depende de que el estado establezca estándares altos y claramente definidos y aumente su capacidad para implementarlos.

La OECD ayuda también a entender la racionalidad que está detrás de focalizarse en la calidad educativa más que en la corrección de un diseño institucional de tanta presencia privada. Para ello, lo fundamental es entender que la calidad educativa forma parte de un esquema más amplio de redefinición de la relación entre lo público y lo privado y, por tanto, de la relación entre Estado y mercado. El contexto general lo constituye la necesidad de reformar y modernizar al Estado de manera que el mismo pueda ayudar al fortalecimiento de los mercados apoyando la competencia económica y el intercambio comercial; todo lo cual tiene incidencia directa sobre la calidad de vida de los países pertenecientes a la OECD (Charbit; Vammalle, 2010; OECD,2005). Esta reforma implica una mejora en el desempeño, eficiencia y trasparencia de la administración pública para acomodarla a las demandas de la economía y los cambios tecnológicos. Mirada desde la educación, también implica que el Estado asuma allí un nuevo rol. Por tanto, corresponde a los gobiernos diseñar programas de calidad que, entre otros aspectos, tengan un sistema de control y medición que aseguren la enseñanza de las habilidades requeridas para que los individuos se integren a la nueva sociedad basada en el conocimiento (OECD, 2012).

Por tanto, la calidad de la educación cumple un rol administrativo no solo orientado a los establecimientos educacionales sino también a re-situar el rol del estado en la educación, subordinando su quehacer a las demandas del mercado. Como lo plantean Olssen y Peters (2005, p. 319), esta nueva forma de gobierno constituye una forma de "gobernar sin gobernar". En relación a los sistemas educativos, se refiere a un proceso por el cual los estados "pilotean a la distancia" de manera a no estorbar el protagonismo del mercado, mientras se crean las condiciones para que el mismo opere normalmente (Ball, 1994; Hursh, 2005). En definitiva, se trata de que los gobiernos sepan intervenir de manera apropiada para resolver aquellas dificultades que terminan comprometiendo la relación entre sistema escolar y economía. 
En ese sentido, incluso para revertir o regular la privatización de los establecimientos, el estado debe hacerlo conforme a una racionalidad en base a la calidad educativa puesto que es la forma adecuada para no entorpecer el desarrollo del sistema conforme a los desafíos de la economía global en donde la competencia entre instituciones y la privatización siguen ocupando un rol central.

\section{LA CALIDAD DE LA EDUCACIÓN COMO PARTE DE LA SEGREGACIÓN}

En la literatura sobre la educación en el país, el concepto de capital también ha sido aplicado a la cultura de las personas en consonancia con la teoría del capital humano (Beyer, 2005; Martinic, 2003; Raczynski; Muñoz, 2007). Sin embargo, los autores no siempre desarrollan su entendimiento sobre la expresión. Aun así, en el contexto en que aplican el término "capital cultural", generalmente se refiere a: los contextos de pertenencia de las personas; el alto nivel de presencia de este capital entre personas de altos ingresos y su poca presencia entre personas con bajos ingresos económicos y la importancia de que las personas de bajos recursos económicos puedan aumentar este capital, como lo hacen los grupos más adinerados, lo que les permitiría aumentar sus rendimientos en las escuelas. De acuerdo a Eyzaguirre (1999), fue E. D. Hirsh quien acuñó el término de "capital cultural", una analogía del concepto de capital, para referirse a una cultura común que todo ciudadano promedio debiera tener para sacar provecho de la sociedad. En consonancia con ello, Eyzaguirre (2004) afirma que el currículo chileno debería entregar los conocimientos que priman en la cultura dominante y que están ausentes entre los grupos con menos recursos.

La referencia de Eyzaguirre (1999) a Hirsh es significativa, puesto que la crítica dirigida a este autor puede aplicarse también a muchas de las recomendaciones sobre la educación en Chile. Por ejemplo, Feinberg (1997) critica el uso que Hirsh hace del concepto "capital cultural" que tomó del trabajo de Bourdieu, representante teórico por excelencia de este término. En lo fundamental, para Bourdieu (1984), el capital cultural ayuda a la creación de riqueza pero predominantemente en medio de grupos afluentes. Como complementan Lareau y Horvat (1999), aunque el capital cultural puede otorgar algunas posibilidades a grupos de escasos recursos, mayormente tiende a la perpetuación de jerarquías sociales desiguales en base a diferencias de clase y raza. Por tanto, la perspectiva del capital cultural desde Bourdieu (1984), más que ayudar a entender cómo se puede elevar los recursos económicos de los diversos grupos sociales, ayuda a comprender cómo se produce la acumulación a nivel cultural, y como consecuencia de ello, la segregación en base a la distinción.

Atria (2012) es probablemente quien mejor sintetiza este proceso de segregación en Chile que ha resultado de prácticas que se definen como centradas en el aumento de la calidad de la educación y del capital cultural de los estudiantes. En su descripción, el autor plantea que los establecimientos educativos y los padres o apoderados, siguiendo la lógica del mercado, se eligen mutuamente por medio de una oferta educativa que contempla que los estudiantes no se mezclarán con otros "que estén en peores condiciones que ellos" puesto que perjudicaría tanto su 
desempeño académico como la calidad de la educación del establecimiento mismo (Atria, 2012, p. 46). Replicado este principio a lo largo de todo el sistema educativo, el resultado final es que "tras la segregación por clase, en la educación pública de establecimientos no 'emblemáticos' queda el gueto de los que no tienen libertad para elegir porque no satisfacen criterio alguno de selección" (idem, p. 40). Este "poder de elegir" apunta a un aspecto esencial del capital cultural de Bourdieu que se visibiliza por medio de la práctica de la selección en el sistema.

Existen diversos estudios sobre la selección en Chile para elevar la competencia entre establecimientos educativos (García-Huidobro, 2007; McEwan, 2001,Parry,1996). En general, estos estudios plantean que la desigualdad en "calidad educativa" reflejada en puntajes SIMCE disminuye entre establecimientos privados y públicos si se toman en consideración las variables económicas y sociales. Por tanto, las diferencias brutas en puntajes que aparecen entre estos establecimientos se deben principalmente a las prácticas de los privados en seleccionar a los alumnos con mejor perfil académico y económico concentrando, así, los mejores resultados. El otro rostro de este proceso lo constituyen los padres y apoderados que usan su capital cultural acumulado y representado por el potencial SIMCE de sus hijos para elegir y ser elegidos por colegios que los segreguen de grupos sociales percibidos como amenaza a sus intereses y que se encuentran a medida que va bajando la escala social y económica. Esta mayor concentración de homogeneidad de clase se encuentra en los colegios privados de elite (Mizala; Romaguera, 2000). Estos colegios atienden a los grupos más adinerados, transformados en referencia indiscutida de concentración de capital económico, asociada a segregación residencial, en donde, además, se ubican estos colegios a los que asisten sus hijos (Cavieres Fernández, 2009a).

El comportamiento de los padres en torno al poder elegir y ser elegidos por los establecimientos educativos ha sido confirmado igualmente por diversos estudios. De acuerdo al estudio de Hsieh y Urquiola (2006, p. 1.479), "el principal efecto de una libertad sin restricción para elegir entre escuelas fue un éxodo de la clase media del sector publico [...] y la pérdida de esos estudiantes tuvo un efecto enorme en el sector público". El estudio de Schneider, Elacqua e Bucley (2006) complementa esta conclusión, señalando que los padres de la región metropolitana, en donde se ubica la capital del país, acentúan más las variables demográficas que las académicas para la selección del centro educativo para sus hijos. En otras palabras, los padres eligen establecimientos en donde sus hijos estudien con pares que pertenezcan a su mismo nivel socioeconómico. Las causas de esta segregación se podrían deber a la libertad de los establecimientos educativos privados de elegir a aquellos estudiantes que son académicamente y económicamente más rentables y a la voluntad de los padres de evitar enviar a sus hijos con alumnos de clase económica inferior bajo el supuesto que los alumnos de clase económica más elevada rinden mejor académicamente como se refleja en el SIMCE.

Un buen resumen del foco del debate que con posterioridad se ha suscitado sobre el tema de la selección, lo ilustra Contreras (2010) cuando sugiere que si el propósito de las modificaciones integradas recientemente a la Ley General de Educación, que guía al sistema chileno, es elevar la calidad de la educación del país, 
"la pregunta debe ser aquí si la selección es buena o mala-adecuada o no-en relación a ese propósito" (Contreras, 2010, p. 322). En ese sentido, las diversas aproximaciones al tema -con las debidas discrepancias respecto a los efectos positivos o negativos de la elección -excluyen en su análisis a la calidad de la educación como una variable clave sobre la cual se establece la selección, y más bien la proyectan dentro de un horizonte en el cual, si la aspiración final es que todos los niños alcancen altos resultados de aprendizaje, esta calidad resolverá cualquier segregación grave (Beyer, 2007; Mena; Corbalán, 2010; Sapelli, 2006). En otras palabras, el SIMCE refleja las desigualdades pero no las produce. Por lo contrario, si los mecanismos de calidad educativa se aplicaran adecuadamente, las diferencias en los logros de aprendizaje disminuirían.

No obstante, dicha aproximación soslaya las implicancias que tiene la calidad de la educación en cuanto forma de capital cultural. Este aspecto, ausente en la literatura chilena, ha sido abordado en los análisis de reformas educativas en países anglos-sajones. En primer lugar, como lo explica Apple (1996) el régimen de exámenes -elemento nuclear de la calidad de la educación-, constituye un elemento esencial para cualquier forma de marketización en la medida en que ofrece los datos necesarios para los diversos análisis comparativos que se requieren para hacer funcionar un mercado. En segundo lugar, los instrumentos usados para la medición de la calidad educativa constituyen un mecanismo de reproducción de clase al ser promovidos e implementados por grupos sociales a quienes las relaciones de mercado les resultan familiares y cómodas, considerando su contexto social y cultural que, unido a los recursos económicos que disponen, les permiten optimizarlas para provecho personal (Apple, 2001; Ball; Bowe; Gewirtz, 1994; Whitty, 2002). Como lo sintetiza Apple (2004, p. 22), siguiendo a Bourdieu, "este sentido de lo que podríamos llamar 'confianza' es el capital invisible que subyace a [1a] capacidad de negociar con las formas marketizadas".

Desde esa perspectiva, la calidad de la educación constituye una forma de capital cultural que contiene una suerte de connaturalidad con quienes culturalmente tienen afinidad con ella por estar habituados a su sistema de medición y puntaje debido a su locación social y económica. Los puntajes que obtiene en pruebas estandarizadas no vienen más que a reforzar una vinculación ya existente que le permite al individuo elegir y ser seleccionado (colegios, universidades, lugares de trabajo, etc.) para seguir avanzando en su movilidad social y económica. Además, en la medida en que el mercado no exige ninguna forma de solidaridad social, los individuos pueden avanzar en esta movilidad precisamente sobre la base de distinguirse respecto a quienes no poseen el mismo capital cultural (o "aprendizajes"), lo que en buena parte está determinado a nivel social por la redistribución desigual de recursos materiales y simbólicos. De este modo, el capital cultural se transforma en factor de competencia que beneficia únicamente a quienes están preparados para competir. Como concluye Bourdieu (1984), en ese contexto, los estudiantes que provienen de contextos con menos recursos económicos están vencidos antes de que la competencia comience. 


\section{LA CALIDAD DE LA EDUCACIÓN COMO PARTE DE LA EXCLUSIÓN}

A las críticas ya reseñadas respecto al sentido otorgado a la noción de capital cultural como cultura dominante a la que deben acceder todos los estudiantes (Eyzaguirre, 1999), se le pueden unir otras de carácter exclusivamente cultural. Según McLaren (1988), el sitial que en esa perspectiva se le otorga a una cultura dominarte conlleva el peligro de situar como "culturalmente deficientes" a quienes están fuera de ella. Igualmente, Buras (2006, p. 49) advierte que dicha aproximación conlleva una connotación no reconocida de "supremacía cultural". Como lo recuerda Delpit (1988), los códigos predominantes en la escuela son arbitrarios en cuanto sólo reflejan aquellos grupos con mayor poder en la sociedad. Si estudiantes de bajos recursos económicos han de acceder a esos códigos, no deben hacerlo ignorando la sapiencia que proviene de sus propios contextos culturales. No obstante, existe abundante literatura que advierte que la aplicación de mediciones estandarizadas desplaza la incorporación de contextos culturales que son relevantes para los alumnos y que también deberían ser integrados dentro de las prácticas pedagógicas en el aula (Kesson, 2008; Linné, 2001).

En América Latina el desplazamiento de determinadas formas culturales por otras dominantes se ha realizado fundamentalmente por acentuar algunos modelos curriculares por sobre otros para alinear a las escuelas con el capital cultural y humano que representan las mediciones estándares. De acuerdo a ello, las reformas educativas se han abocado a promover un modelo de buenas prácticas de enseñanza que produzcan los resultados esperados. El programa de Promoción de la Reforma Educativa de América Latina y el Caribe (PREAL), apoyado por la Agencia de los Estados Unidos para el Desarrollo Internacional (USAID) y el Banco Interamericano de Desarrollo (BID), entre otros, es una de las organizaciones que han promovido esta noción de buenas prácticas en países latinoamericanos (Andraca, 2003). La Organización de las Naciones Unidas para la Educación, la Ciencia y la Cultura (UNESCO) también ha promovido este concepto de "buenas prácticas" unido a nociones de eficiencia y resultado de aprendizajes (Blanco, 2008; Mella, 2002).

Una concreción de esta perspectiva en Chile es la promoción de las llamadas escuelas efectivas con apoyo de Fondo de las Naciones Unidas para la Infancia (UNICEF) y el Ministerio de Educación Chileno, cuya finalidad es aumentar el capital cultural de los estudiantes de escasos recursos económicos (Belleï et al., 2004). Este modelo de escuelas enraizado en el análisis escolar realizado en Estados Unidos (Fernández, 2004) -siendo el trabajo de Chubb y Moe (1990) el ejemplo más representativo- ha sido avanzado por diversos investigadores chilenos (Arancibia, 1992; Brunner; Elacqua, 2004; Eyzaguirre; Fontaine, 2008; Raczynski; Muñoz, 2007). De acuerdo a Martinic y Pardo (2002, p. 98), esas investigaciones tienen el objetivo de aprender cómo estas escuelas efectivas logran hacer que sus estudiantes alcancen "metas educativas de calidad independientemente de su origen social". Un ejemplo reciente de esta línea investigativa en Chile lo constituye el estudio sobre escuelas subvencionadas de la ciudad de Santiago y pertenecientes a la Sociedad de Instrucción Primaria que atiende a estudiantes de sectores de bajos recursos 
económicos y que en el tiempo han mejorado sus puntajes SIMCE (Henríquez et al., 2012). Conforme este estudio, dichos resultados en estas escuelas se explican por la estrategia de focalizar la administración, las prácticas pedagógicas, la relación con la comunidad y el aprendizaje de los estudiantes alrededor de los desempeños académicos tal como son medidos por SIMCE.

Sin embargo, este modelo que pretende integrar a los sectores con escasos recursos económicos a una cultura con alto capital humano fomenta una particular forma de exclusión. Cavieres Fernandez (2009b, 2011) ha planteado que la enseñanza de estudiantes de escasos recursos económicos con dicho énfasis ha tendido a concentrarse exclusivamente en los problemas "sociales" que esos alumnos experimentan en sus contextos de origen, ya que no les permite aumentar sus aprendizajes medibles. Estos problemas, además, son vistos primordialmente como obstáculos para que los alumnos y sus apoderados asuman un rol más protagónico en la cultura gerencial y centrada en resultados que se intenta establecer en las escuelas. Esta perspectiva es compartida por Rambla (2006, p. 359) cuando plantea que las implementaciones educativas integradas a la educación en Chile han sido un intento por trasplantar "las reglas sociales de la empresa y el éxito individual desde el sector profesional privilegiado a los sectores populares en desventaja”. En ese contexto, como complementa Rambla y Verger (2009, p. 472), en la medida en que los estudiantes de bajos recursos económicos no responden adecuadamente a estas nuevas reglas, se les ha venido a caracterizar como un "grupo subordinado, pasivo y débil".

En ese contexto es posible situar algunas experiencias que indican el desplazamiento y, por tanto, la exclusión en el currículo de determinadas preferencias culturales de los estudiantes. Por ejemplo, algunas experiencias de educación popular fueron introducidas inicialmente a comienzos de los 1990 en programas de apoyo a estudiantes de bajos recursos económicos. Esta forma de educación tenía larga tradición en Chile (García-Huidobro; Sotomayjor, 1989; Austin, 2003) y, de acuerdo a Duarte (1993), era aceptada por determinados grupos de jóvenes populares porque respetaba sus identidades y se comprometía políticamente con los problemas de sus comunidades. Aunque esas experiencias de educación popular fueron contextualizadas bajo un nuevo marco de indicadores de aprendizajes, igualmente promovieron avances en los jóvenes referidos a su retención y puntajes SIMCE (García-Huidobro; Sotomayor, 2003). Sin embargo, a medida en que se acentuaron aún más los objetivos de la calidad de la educación, y bajo la presión de que los establecimientos públicos debían aumentar más sus puntajes, las estrategias de educación popular fueron dejadas de lado mientras se acentuaba exclusivamente el foco en los aprendizajes en matemáticas y lenguaje (Gysling, 2003).

Diversas cifras también apuntan hacia la exclusión de determinados objetivos educativos ante los requerimientos de la calidad educativa. En general, algunos estudios han apuntado a cierta tensión entre dos modelos educativos que tienden a repartirse la preferencia de los estudiantes chilenos (Arancibia, 1994; Centro de Investigación y Desarrollo de la Educación, 2010). Por un lado, se encuentra en el país lo que se ha venido a llamar una educación integral relacionada a una formación valórica, mientras, por el otro lado, está una educación de calidad referida a una 
formación cognitiva y orientada tanto al mundo del trabajo como a estudios de educación superior. Dado el énfasis de las políticas públicas en el capital humano, es previsible que los estudiantes se interesen por una educación de calidad que les abra expectativas laborales y económicas, a la vez que relacionen la educación con el objetivo general de llegar ser "buenas" personas, lo que tiene un claro contenido valórico.

No obstante, cuando se analiza la distribución horaria de la jornada escolar, se observa que, tomando como referencia el currículo para estudiantes de tercer año de enseñanza media, en promedio se destina alrededor del 55\% del tiempo para lenguaje, matemáticas, historia y ciencias -disciplinas que son consideradas prioritarias para las mediciones estándares en el país. Ese porcentaje aumenta si se consideran los talleres de reforzamiento para estos sectores del aprendizaje y de preparación para los exámenes estándares, y que ocupan parte de las horas que los establecimientos cuentan para organizar actividades de su libre elección (Dirección de Estudios Sociológicos, 2005). Este cálculo porcentual también aumenta si se incluyen las horas que se agregan hacia el final de la enseñanza secundaria destinadas a los planes curriculares diferenciados que profundizan estas disciplinas centrales para los exámenes estándares del currículo; y si se incluyen las horas para la enseñanza del inglés que se justifica como un conocimiento indispensable para integrarse a los procesos de globalización mundial y para poder avanzar en los estudios universitarios o perspectivas laborales (Ministerio de Educación, 2009).

El trabajo de Oyarzún et al. (2001), realizado con jóvenes de clase media y baja, ayuda a contextualizar estas cifras. Entre otros, estos jóvenes privilegian aspectos valóricos como el compañerismo y la solidaridad por sobre la competitividad y muestran alto interés por el arte, la música y el cambio social. Consultados respecto a sus centros educativos, estos jóvenes solicitan tener más espacios de recreación, que se les respete sus derechos y sólo en tercer lugar mencionan mejorar la calidad educativa con una preferencia similar a la de mejorar el diálogo en torno a sus intereses. De su análisis, Oyarzún et al. (idem, p. 222) concluyen que la matriz que se utilizó para mejorar la educación en Chile ha terminando marginando a los estudiantes, dejándolos sin los espacios para "impactar en el aula con sus saberes y sus quehaceres". Desde esa perspectiva, la calidad educativa de la educación ha contribuido, junto a la exclusión material y social, a la marginación cultural de los estudiantes de escasos recursos económicos. No debe extrañar, entonces, que preguntados por su percepción respecto a la juventud, la mayoría de esos jóvenes se inclinen por retratarla como vulnerable, sin oportunidades de estudio y trabajo, mientras que solo una minoría la reconoce como exitosa y competitiva (idem).

\section{CONCLUSIÓN}

A lo largo de este trabajo se ha discutido que la noción de calidad de la educación constituye -en cuanto racionalidad y sistema formado por mecanismos de fijación y monitoreo del currículo- un elemento central de las implementaciones educativas a nivel mundial y de forma específica en Chile. Por tanto, no puede ser omitido de los análisis críticos que se llevan a cabo con la finalidad de demandar 
mejorías sustantivas en el país, que reviertan los efectos negativos que han resultado de políticas educativas centradas en torno a nociones de crecimiento económico, competencia y de capital humano y cultural. Esa perspectiva concuerda con el análisis realizado por Oliva (2010, p. 325) que plantea que las reformas educativas en Chile constituyen un entramado de elementos todos los cuales confluyen a la alianza entre la "teoría del capital humano y el currículum técnico". Dentro de ese contexto, he situado la noción de calidad educativa como parte de esta trama.

Reconocer la calidad educativa como parte del problema educacional en Chile, que produce una desmedida privatización con la consiguiente segregación y exclusión, obliga a incorporar una mirada sobre el currículo escolar que sea coherente con las demandas más amplias referidas a la igualdad de financiamiento y acceso al sistema educativo. La noción de calidad educativa no garantiza la plena incorporación de los estudiantes a la sociedad, puesto que, más allá de las correcciones que se le puedan añadir, se sustenta en una racionalidad económica que finalmente sitúa el aprendizaje como un producto a ser transado en los mercados competitivos. Desde esa perspectiva, es más consistente vincular las demandas en contra de la privatización del sistema con aquellas corrientes curriculares que, también desde el ámbito internacional, avanzan nociones de democratización y justicia social (Apple; Beane, 2007; Ayers; Klonsky; Lyon, 2000; Meier, 2002). Estas corrientes están en consonancia con tradiciones en Chile y América Latina que, en contextos de desigualdad económica, relacionan la educación con la transformación política y social (Fals-Borda, 1991; Freire, 2000). Esta conexión permitirá ahondar mejor los desafíos y resistencias que proceden desde la educación pública frente a la absolutización de la racionalidad económica en las actuales implementaciones educativas.

\section{REFERENCIAS}

Arancibia, Violeta. La educación en Chile: percepciones de la opinión pública y de expertos. Estudios Públicos, Santiago de Chile: Centro de Estudios Públicos, n. 54, p. 125-151, 1994.

.Efectividad escolar. Un análisis comparado. Estudios Públicos, Santiago de Chile: Centro de Estudios Públicos, n. 47, p. 101-125, 1992.

Andraca, Ana María de. Buenas prácticas para mejorar la educación en América Latina. Santiago de Chile: PREAL, 2003.

Apple, Michael Whitman; Beane, James A. Democratic schools: lessons in powerful education. Portsmouth, NH: Heinemann, 2007.

Apple, Michael Whitman. Cultural politics and education. New York: Teachers College Press, 1996.

Educating the "right" way: markets, standards, God and inequality. New York: Routledge-Falmer, 2001. 
Creating difference: neo-liberalism, neo-conservatism and the politics of educational reform. Educational Policy, Boston: Boston College; Sage Journal; Corwin Press, v. 18, n. 1, p. 12-44, enero/mayo 2004.

Arellano, José Pablo Políticas sociales para el crecimiento con equidad: Chile 1990-2002. Santiago, Chile: CIEPLAN, 2005.

Atria, Fernando. La mala educación. Ideas que inspiran al movimiento estudiantil en Chile. Santiago de Chile: Catalonia Libros; CIPER, 2012.

Austin, Robert. The state, literacy, and popular education in Chile, 1964-1990. Lanham, Md: Lexington Books, 2003.

Ayers, William; Klonsky, Michael; Lyon, Gabrielle. A simple justice: the challenge of small schools. New York: Teachers College; Columbia University, 2000.

Ball, Stephen. Education reform. A critical and post-structural approach. Philadelphia: Open University Press, 1994.

Ball, Stephen; Bowe, Richard; Gewirtz, Sharon. Market forces and parental choice. In: Tomlinson, Sally (Ed.). Educational reform and its consequences. London: IPPR; Rivers Oram Press, 1994. p. 13-25.

BECKER, Gary S. Human capital: a theoretical and empirical analysis, with special reference to education. New York: National Bureau of Economic Research, 1964.

Belleï, Cristian. Expansión de la educación privada y mejoramiento de la educación en Chile. Revista Pensamiento Educativo, Santiago de Chile: Pontificia Universidad Católica de Chile, v. 40, n. 1, p. 285-311, 2007.

Belleï, Cristián; Muñoz, Gonzalo; Pérez, Luz María; Raczynski, Dagmar. ¿Quién dijo que no se puede?: escuelas efectivas en sectores de pobreza. Santiago de Chile: UNICEF, 2004.

Beyer, Harald; Eyzaguirre, Bárbara; Fontaine, Loreto. La reforma educacional chilena: una apreciación crítica. Santiago, Chile: Centro de Estudios Públicos, 2000.

Productividad, desigualdad y capital humano: los complejos desafíos de Chile. Estudios Públicos, Santiago de Chile: Centro de Estudios Públicos, n. 97, p. 59-98, 2005.

- ¿Qué hacer con la educación pública? Estudios Públicos, Santiago de Chile: Centro de Estudios Públicos, n. 114, p. 89-125, 2009.

. Algunas notas sobre la selección educacional. In: Brunner, José Joaquim; Peña, Carlos (Coords.). La reforma al sistema escolar: aportes para el debate. Santiago de Chile: Universidad Diego Portales; Universidad Adolfo Ibáñez, 2007. p. 221-238.

Blanco, Rosa. Eficacia escolar y factores asociados en América Latina y el Caribe. Santiago de Chile: UNESCO-LLECE, 2008.

Bourdieu, Pierre. Distinction. A social critique of the judgment of the taste. Cambridge, MA: Harvard University Press, 1984.

Brunner, José Joaquim; Elacqua, Gregory. Informe capital humano en Chile. Santiago, Chile: Universidad Adolfo Ibañez; La Araucana, 2003.

Factores que inciden en una educación efectiva. Evidencia Internacional. $\mathrm{La}$ Educación, OEA, n. 139-140, p. 1-11, 2004. 
PeÑa, Carlos. Introducción. In: .(Coords.). La reforma al sistema escolar: aportes para el debate. Santiago de Chile:Universidad Diego Portales; Universidad Adolfo Ibáñez, 2007.p. 9-22.

Buras, Kristen. Tracing the core knowledge movement: history lessons from above and below. In: Apple, Michael Whitman; Buras, Kristen (Eds.). The subaltern speak. Curriculum, power, and educational struggles. New York: Routledge, 2006. p. 43-74.

Cavieres Fernández, Eduardo. Rethinking the role of elite private schools in a neoliberal era. An example from Chile. Policy Futures in Education, Oxford, Reino Unido: Symposium Journals, v. 7, n. 1, p. 31-43, 2009a.

La neoliberalización de la educación chilena o la privatización del sistema educativo por "dentro". Revista Electrónica Diálogos Educativos, Santiago de Chile: Universidad Metropolitana de la Educación, año 9, n. 17, p. 18-57, 2009b.

. The class and culture-based exclusion of the chilean neoliberal educational reform. Educational Studies, Nueva York: AESA; Routledge, v. 47, n. 2, p. 111-132, 2011. Centro de Investigación y Desarrollo de la Educación. VII Encuesta Cide a actores del sistema educativo. Santiago de Chile: Centro de Investigación y Desarrollo de la Educación; Universidad Alberto Hurtado, 2008.

VIII Encuesta CIDE a actores del sistema educativo. Santiago de Chile: Centro de Investigación y Desarrollo de la Educación; Universidad Alberto Hurtado, 2010.

Charbit, Claire;Vammalle, Camila. Modernizing government. In: OECD (Ed.). Making reform happen. Lessons from OECD countrie. Paris: OECD Publishing, 2010. p. 209-238.

Снивв, John E.; Moe, Terry M. Politics, markets, and America's schools. Washington, DC: Brookings Institution Press, 1990.

Contreras, Daniel. Derecho a la educación, inclusión y selección escolar. In: Belleï, Cristian; Contreras, Daniel; Valenzuela, Juan Pablo (Eds.). Ecos de la revolución pinguina. Avances, debates y silencios en la reforma educativa. Santiago de Chile: UNICEF; Universidad de Chile, 2010. p. 311-330.

Cornejo, Rodrigo. El experimento educativo chileno 20 años después: una mirada crítica a los logros y falencias del sistema escolar. Revista Electrónica Iberoamericana sobre Calidad, Eficacia y Cambio en Educación, Madrid: RINACE, v. 4, n. 1, p. 118-129, 2006.

Departamento de Estudios y Desarrollo. Documento estadísticas de la educación en Chile 2009. Santiago, Chile: Ministerio de Educación; Gobierno de Chile, 2010.

Dirección de Estudios Sociológicos. Informe final. Evaluación Jornada Escolar Completa. Santiago de Chile: Pontificia Universidad Católica de Chile; Dirección de Estudios Sociológicos, 2005.

DelPit, Lisa. The silenced dialogue: power and pedagogy in educating other people's children. Harvard Educational Review, Cambridge, MA: Harvard Graduate School of Education; Harvard Education Publishing Group, v. 58, n. 3, p. 280-298, 1988.

Duarte, Klaudio. Juventud popular. El rollo entre ser lo que queremos o ser lo que nos imponen. Santiago de Chile: LOM Ediciones, 1993. 
Eyzaguirre, Bárbara. Una mirada a la reforma curricular. Estudios Públicos, Santiago de Chile: Centro de Estudios Públicos, n. 76, p. 263-296, 1999.

Claves para la educación en pobreza. Estudios Públicos, Santiago de Chile: Centro de Estudios Públicos, n. 93, p. 249-277, 2004.

Eyzaguirre, Bárbara; Fontaine, Loreto. Las escuelas que tenemos. Santiago, Chile: Centro de Estudios Públicos, 2008.

Fals-Borda, Orlando. Knowledge and social movements. Santa Cruz, CA: Merrill Publications; University of California, Santa Cruz, 1991.

FeInberg, Walter. Educational manifestos and the new fundamentalism. Educational Researcher, Nueva York: AESA; Sage Journals, v. 26, n. 8, p. 27-35, 1997.

Fernández, Tabaré. De las "escuelas eficaces" a las reformas educativas de "segunda generación”. Estudios Sociologicos, Distrito Federal, México: El Colegio de México, v.XXII, n. 2, p. 377-408, 2004.

Freire, Paulo. Pedagogy of the oppressed. New York: Continuum, 2000.

García-Huidobro, Juan Eduardo. La propuesta pedagógica de los proyectos de educación popular. In: García-Huidobro, Juan Eduardo; Martinic, Sergio; Ortiz, Iván (Eds.). Educación popular en Chile. Trayectoria, experiencias y perspectivas. Santiago de Chile: Centro de Investigación y Desarrollo de la Educación, 1989. p. 75-110.

. La "selección de los alumnos" en la ley general de la educación. Revista Docencia, Santiago de Chile: Colegio de Profesores de Chile A. G., n. 32, p. 20-27, 2007.

Sotomayor, Carmen. La centralidad de la escuela en la política educativa chilena de los años noventa. In: Cox, Cristian (Ed.). Políticas educacionales en el cambio de siglo: la reforma del sistema escolar de Chile. Santiago de Chile: Editorial Universitaria, 2003. p. 253-316.

Gobierno de Chile. Sintesis de resultados. SIMCE 2011. Gobierno de Chile; Sistema de Medición de Calidad de la Educación, 2012.

GonzÁlez, Pablo. The challenge of regulating private education. In: WolfF, Laurence; Navarro Juan Carlos; González, Pablo (Eds.). Private education and public policy in Latin America. Washington, DC: PREAL, 2005. p. 227-236.

Gutierrez, Tamara; Caviedes, Cristina. Revolución pingüina: la primera gran movilización del siglo XXI en Chile. Santiago de Chile: Ayun, 2006.

Gysling, Jacqueline. Reforma curricular: itinerario de una transformación cultural. In: Cox, Cristian (Ed.). Politicas educacionales en el cambio de siglo: la reforma del sistema escolar de Chile. Santiago, Chile: Editorial Universitaria, 2003. p. 213-252.

Henrí́uez, Francisco; Lara, Bernardo; Mizala, Alejandra, Repetto, Andrea. Effective school do exist: low income children's academic performance in Chile. Applied Economic Letters, Nueva York: Routledge, v. 19, n. 5, p. 445-451, 2012.

HiLl, Paul T. The educational consequences of choice. Phi Delta Kappan, Bloomington: Phi Delta Kappan International, v. 77, n. 10, p. 671-676, 1996.

HoxBy, Caroline M. Do private schools provide competition for public schools? NBER Working Paper Series, n. 4.978. Cambridge, MA: NBER, 1994. p. 1-55. 
Hsien, Chang-Tai; Urquiola, Miguel. The effects of generalized school choice on achievement and stratification: evidence from Chile's voucher program. Journal of Public Economics, Elsevier, v. 90, n. 8-9, p. 1.477-1.503, 2006.

Hursh, David. Neo-liberalism, markets and accountability: transforming education and undermining democracy in the United States and England. Policy Futures in Education, Oxford, Reino Unido: Symposium Journals, v. 3, n. 1, 2005.

Kesson, Kathleen. Teaching. In: Gabbard, David (Ed.). Knowledge and power in the global economy: the effects of school reform in a neoliberal/neoconservative age. New York: Lawrence Erlbaum Associates, 2008.

Lareau, Anette; Horvat, Erin. Moments of social inclusion and exclusion. Race, class, and cultural capital in family-school relationships. Sociology of Education, Washington, DC: American Sociological Association; Thousand Oaks, CA: Sage Publications, v. 72, n. 1, p. 37-53, 1999.

Linné, Rob. Teacher perspectives on standards and high-stakes testing. From the urban to the suburban. In: Kincheloe, Joe L.; Weil, Danny K. (Eds.). Standards and schooling in the United States: an encyclopedia. Santa Barbara, CA: ABC-CLIO, 2001.v. 3, p.1.083-1.096. Martinic, Sergio; PARdo, Marcela. Aportes de la investigación educativa iberoamericana para el análisis de la eficacia escolar. In: Murillo, F. Javier. La investigación sobre eficacia escolar en Iberoamérica. Revisión internacional sobre estado del arte. Bogotá: Convenio Andrés Bello; Ministerio de Educación, Cultura y Deporte, España y CIDE, 2002. p. 93-125.

Martinic, Sergio. Las representaciones de la desigualdad en la cultura escolar. Persona y Sociedad, Santiago de Chile: Universidad Alberto Hurtado, XVII, n. 1, p. 129-145, 2003. McEwan, Patrik J. The effectiveness of public, catholic, and non-religious private schools in Chile's voucher system. Education Economics, Nueva York: Routledge, v. 9, n. 2, p.103$-128,2001$.

McLaren, Peter. Culture or canon? Critical pedagogy and the politics of literacy. Harvard Educational Review, Cambridge, MA: Harvard Graduate School of Education; Harvard Education Publishing Group, v. 58, n. 2, p. 213-234, 1988.

MeIER, Deborah. In schools we trust: creating communities of learning in an era of testing and standardization. Boston: Beacon Press, 2002.

Mella, Orlando. Estudio cualitativo de escuelas con resultados destacables en siete países Latinoamericanos. Santiago de Chile: UNESCO; LLECE, 2002.

Mena, Paula; Corbalán, Francisca. La selección escolar: una cuestión de libertad, competencia, igualdad e integración. In: Belleï, Cristian; Contreras, Daniel; Valenzuela, Juan Pablo (Eds.). Ecos de la revolución pinguïna. Avances, debates y silencios en la reforma educativa. Santiago de Chile: UNICEF; Universidad de Chile, 2010. p. 331-358. 
Ministerio de Educación. Evaluación de aprendizajes para una educación de calidad. Santiago de Chile: Comisión para el Desarrollo y Uso del Sistema de Medición de la calidad de la Educación; Ministerio de Educación, 2003.

. Objetivos fundamentales y contenidos minimos obligatorios de la educación básica y media. Santiago de Chile: Ministerio de Educación, 2009.

Mizala, Alejandra; Romaguera, Pilar. School performance and choice. The Chilean experience. The Journal of Human Resources, Madison, WI: The University of Wisconsin Press, v. 35, n. 2, p. 392-417, 2000.

.La subvención escolar diferenciada por nivel socioeconómico en Chile. In: BELLEÏ, Cristian; Contreras, Daniel; Valenzuela, Juan Pablo (Eds.). La agenda pendiente en educación: profesores, administradores y recursos: propuesta para la nueva arquitectura de la educación chilena. Santiago: Universidad de Chile, 2008.

OECD. Reviews of national policies for education. Chile; Paris: OECD Publishing, 2004. Modernizing government. The way forward. Paris: OECD, 2005.

Maintaining momentum: OECD perspectives on policy challenges in Chile. Paris: OECD Publishing, 2011.

. Better skills, better jobs, and better lives. A strategic approach to skills policies. OECD Publishing, 2012.

Oliva, María Angélica (2010). Política educativa chilena: 1965-2009. ¿Qué oculta esta trama? Revista Brasileira de Educação, Rio de Janeiro: ANPEd; Campinas: Autores Associados, v. 15, n. 44, p. 311-328, mayo/ago. 2010.

Olssen, Mark; Peters, Michael. Neoliberalism, higher education and the knowledge economy: from the free market to knowledge capitalism. Journal of Education Policy, Nueva York: Routledge, v. 20, n. 3, p. 313-345, 2005.

Oyarzún, Astrid; Irrazabal, Raúl; Goicovic, Igor; Reyes, Leonora. Entre jóvenes reproductores y jóvenes co-constructores: sentidos de la integración en la cultura escolar. Viña del Mar: CIDPA Ediciones, 2001.

PARRY, Taryns R. Will pursuit of higher quality sacrifice equal opportunity in education? An analysis of the education voucher system in Santiago, Social Science Quarterly, Hoboken, NJ: Southwestern Social Science Association, v. 77, n. 4, p. 821-841, 1996.

Raczynski, Dagmar; MuÑoz, Gonzalo. Factores que desafían los buenos resultados educativos de escuelas en sectores de pobreza. In: Cueto, Santiago (Ed.). Educación y brechas de equidad en América Latina. Santiago, Chile: PREAL, 2007. Tomo II, p. 275-352.

Rambla, Xavier. Globalization, educational targeting, and stable inequalities: acomparative analysis of Argentina, Brazil, and Chile. International Review of Education, Hamburg: UNESCO Institute for Lifelong Learning; New York: Springer, v. 52, n. 3-4, p. 353-370, 2006.

Veger, Antoni. Pedagogising poverty alleviation: a discourse analysis of educational and social policies in Argentina and Chile. British Journal of Sociology of Education, Nueva York: Routledge, v. 30, n. 4, p. 463-477, 2009. 
Rojas, Jorge. Sociedad bloqueada. Movimiento estudiantil, desigualdad y despertar de la sociedad chilena. Santiago de Chile: Ril Editores; Universidad de Concepción, 2012.

SAmoff, Joel. Which priorities and strategies for education? International Journal of Educational Development, Elsevier, v. 16, n. 3, p. 249-271, 1996.

SApelli, Claudio. Desafíos del sistema educativo: la subvención preferencial. Temas de la Agenda Pública, Santiago de Chile: Pontificia Universidad Católica de Chile, año 1, n. 1, p. 1-8, 2006.

Schneider, Mark; Elacqua, Gregory; Buckley, Jack. School choice in Chile: is it class or the classroom? Journal of Policy Analysis and Management, Association for Public Policy Analysis and Management: Wiley online Library, v. 25, n. 3, p. 577-601, 2006.

Schultz, Theodore. Investment in human capital. The role of education and of research. New York: The Free Press, 1971.

The World Bank. Chile. Secondary education quality improvement Project. Staff appraisal report. Washington, DC: The World Bank, 1995a.

Priorities and strategies for education. A World Bank review. Washington, DC: The World Bank, 1995b.

Torche, Florencia. Privatization reform and inequality of educational opportunity. The case of Chile. Sociology of Education, Washington, DC: American Sociological Association; Thousand Oaks, CA: Sage Publications, v. 78, n. 4, p. 316-343, 2005.

Torres, Carlos Alberto. The state, privatization, and educational policy: a critique of neo-liberalism in Latin America and some ethical and political implications, Comparative Education, Nueva York: Routledge, v. 38, n. 4, p. 365-385, 2002.

Universidad de Chile. Compendio Estadístico Proceso de Admisión Año Académico 2011. Santiago de Chile: Universidad de Chile; Vicerrectoría de Asuntos Académicos; DEMRE, 2011.

Whitty, Geoff.Making sense of education policy. London: Paul Chapman Publishing, 2002. Wolff, Laurence; Moura Castro, Claudio de. Public or private education in Latin America?: asking the wrong question. In: Wolff, Laurence; Navarro, Juan Carlos; GonzÁlez Pablo (Eds.). Private education and public policy in Latin America. Washington, DC: PREAL, 2005.

\section{SOBRE EL AUTOR}

Eduardo Cavieres Fernández es PhD en educación por la University of Wisconsin-Madison (Estados Unidos). Es profesor de la Universidad de Playa Ancha (Chile).

E-mail: ecavieres-cea@upla.cl 
